\title{
Infrared Sights Zero Momentum Measurement Technology based on Camera Adaptive Algorithm

\author{
Jin-Song WANG ${ }^{1, a}$, Dan $\mathrm{HU}^{1, \mathrm{~b}^{*}}$, Yan-Feng $\mathrm{LI}^{1, \mathrm{c}}$ \\ ${ }^{1}$ Changchun University of Science and Technology photoelectrical engineering institute, Jilin, \\ Changchun \\ asoldier_1973@163.com, hohu_9008@163.com, cyannianyishou@126.com \\ ${ }^{*}$ Corresponding author
}

Keywords: Zero Momentum, Measurement, Image Interpretation.

\begin{abstract}
According to the high precision testing requirements on aiming at the baseline variation of infrared sight, a measuring method of infrared sight zero momentum based on camera pose adaptive mathematical model is proposed. A new adaptive algorithm of camera pose is designed. A camera pose correction model is established, which is applied to zero momentum measuring system firstly, and a problem of zero momentum measuring error caused by camera tilting is solved. The pinhole targets and sighting divide image is located by gravity method, and sub-pixel subdivision of edge points is achieved by Zernike orthogonal moments. An experimental system is constructed; the measurement accuracy is superior to $0.02 \mathrm{mil}$ which is verified by experiment, which meets the high precision testing requirement of infrared sight baseline variation.
\end{abstract}

\section{Introduction}

With the rapid development of photoelectric technology and infrared thermal imaging technology, infrared products are getting more and more attention [1]. As night combat mainstream sights, infrared sight has good concealment, can effectively identify camouflage, etc, it's a popular development direction of currently sights series. Infrared sights can give weapon proper shooting, so soldiers can damage enemy targets at the expected position, so the stability of sight baseline is the fundamental guarantee that the infrared sight can achieve its basic mission. However, due to shooting vibration, temperature changes and other factors, easily lead to infrared sight baseline change, impact hit accuracy. Therefore, in order to give full play to the performance of the infrared sights required to detect infrared sight baseline variation [2].

Abroad mainly in the United States OPTIKOS and the Israeli company's CI infrared detection equipment, can detect MRTD, MDTD and MTF indicators of infrared technology, But about the sights aiming baseline variation detection rarely reported [3]. This paper design the method of infrared sight baseline variation, employing a reflective collimator and the image interpretation technology for detection, Get pinhole target and aiming reticle image by area CCD camera, based on Zernike moments invariant properties, round spot on the edge pixels sub pixel, recognition Vipera Center and aiming reticle center by gravity method, Designed a camera posture correction algorithm, Can effectively improve the infrared sight baseline variation detection efficiency and accuracy[4].

\section{Zero Momentum Measurement Principle}

Sight's zero momentum, as the name suggests refers to the sights in environmental testing, after the shooting attack, the value of baseline deviate from the original location. This deviation must be guaranteed within a certain range, otherwise it will affect the accuracy of firearms shooting, Even indirectly restrict the development of light weapons. Fig.1 shows zero momentum measuring principle diagram [5]. 


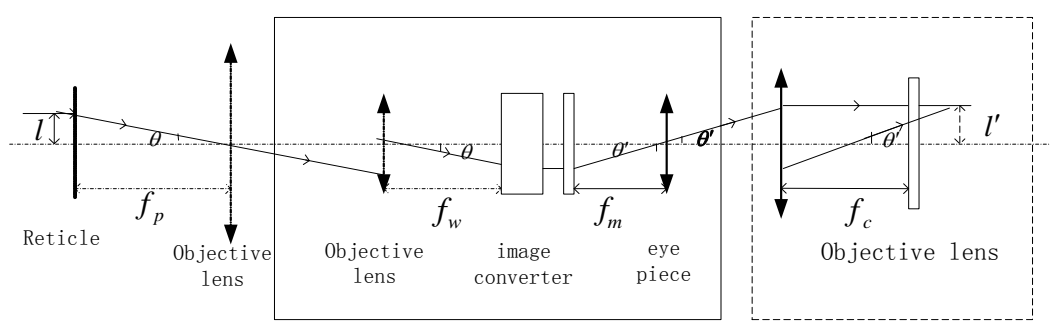

Fig.1 Zero momentum measuring principle diagram

Provided collimator focal length $f_{p}$, subjects sight of the objective focal length $f_{w}$, eyepiece focal length $f_{m}$, CCD camera lens focal length lens for $f_{c}, l$ is CCD camera for the target image height, $l^{\prime}$ is the target height, $l_{b}$ is image height in infrared focal plane arrays sights, calculation by formula(1)

$$
\alpha_{c}=\alpha * \frac{l_{c}^{\prime}}{l}
$$

Wherein, $\alpha$ is an opening angle formed between the target to collimator lens, by 0.5 "Leica theodolite after calibration accuracy for $3.179 \mathrm{mil}, l_{c}^{\prime}$ and $l^{\prime}$ can be measured by the data processing software. By the formula (1) is obtained Infrared sights zero momentum [6].

\section{Measurement Methods to Achieve}

\section{System Components and Working Principle}

The system consists of infrared collimator, CCD cameras, lenses and data processing software. Among them infrared collimator consists axis parabolic mirror, flat mirror, blackbody and pinhole target. As shown in Figure 2 for System Diagram [8].

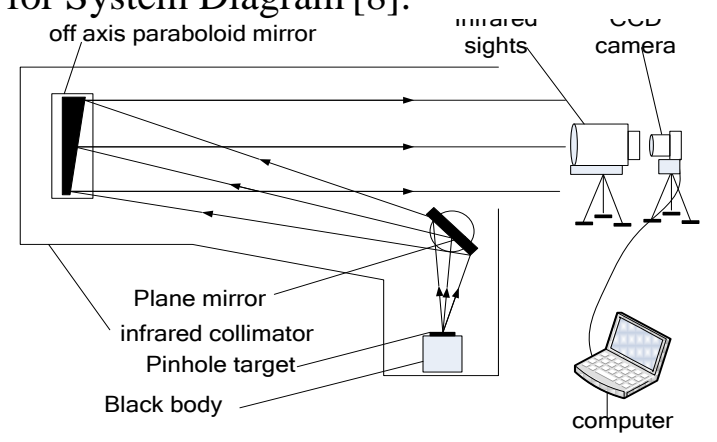

Fig.2 System working principle diagram

Measured infrared sights and camera CCD are respectively disposed in respective mounts, CCD camera lens keep at the exit pupil of sight of the measured sights, Alignment of the main axis of the infrared collimator. Properly adjust the CCD camera lens aperture and focal length, observed a clear pinhole target and aiming partition. According to the collected image, judge and read infrared sights aiming partition coordinate $S\left(x_{s}, y_{s}\right)$ at measuring reference of pinhole target, substituting into equation (2) obtained respectively in the $\mathrm{X}$ direction, $\mathrm{Y}$ direction angle value. 


$$
\begin{aligned}
& X_{s}=\alpha * \frac{x_{s}}{l} \\
& Y_{s}=\alpha * \frac{y_{s}}{l}
\end{aligned}
$$

Remove sights, after the shooting attack, and a series of high and low temperature test again chucking, measuring coordinate partition $\mathrm{T}\left(x_{t}, y_{t}\right)$, ditto, Obtain $X_{t}$ and $Y_{t}$, the difference between the two positions $\Delta \mathrm{X}$ and $\Delta \mathrm{Y}$ is zero momentum of this sights.

$$
\Delta X=X_{t}-X_{\mathrm{s}} \quad \Delta Y=Y_{t}-Y_{\mathrm{s}}
$$

\section{Pinhole Target Infrared Image Recognition}

In this paper, center of gravity method is adopted to identify pinhole target of five circular spot and locate the center coordinates.

First, let entire target pinhole image to median filter, remove noise. To improve the detection efficiency, selected target area by artificial, frame select each round spot as identification zone. Assuming the selected pixel spots on row $i$ and column $j, G[i, j]$ for the pixel gray value of the i-th row j-th column.

Zernike matrix weighted neighborhood edge points by one plurality of templates and two real numbers template, calculate the space matrix, using matrix invariant properties, draw direction angle of edge and distance from the center from the edge of the template 1 and other parameters. Since this selected CCD camera pixel is square, if $|l| \geq \frac{1}{\sqrt{2}}$, believes that the current pixel point does not belong to the circle edge point spots, otherwise, it is determined that the edge point. Using $\varphi$ and $l$ correct the coordinates of round spot edge point, come round spot edge sub-pixel level, thus improving the accuracy of centering round spot.

\section{Model Cameras Posture Adaptive Correction}

In actual measurement, it's difficult to ensure CCD camera not to be disturbed or slight vibration during test environment when chuck infrared sights, easy to lead to changes in posture.

If the camera is tilted, as a reference to the CCD camera, the camera does not move, but the image position of the sub-scribe line and round spot has changed, Figure 3 shows the comparison chart for the CCD camera posture without tilting the camera and tilt angle when small.

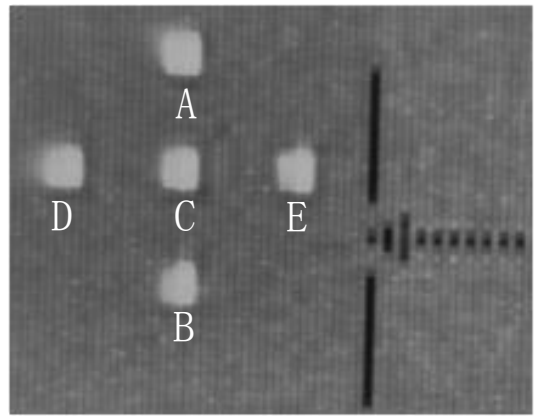

(a) CCD camera without tilting

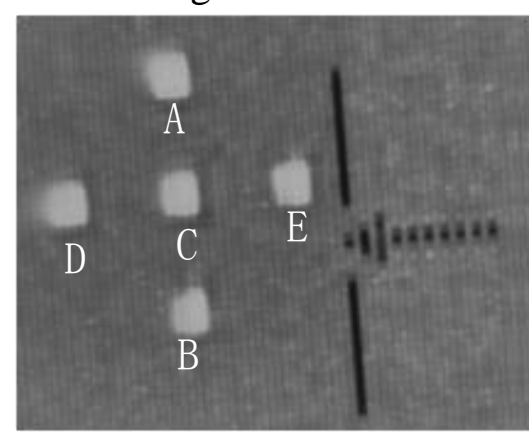

(b) CCD camera tilting

Fig.3 Camera pose comparison diagram

Coordinates A, B, C, D and E point obtained by the image recognition algorithm, figure 6 shows a schematic diagram of a round spot image, In DE abscissa, AB vertical axis, establish a Cartesian coordinate system. 
Successively ergodic search erea, calculate minimum distance squared and counterclockwise and clockwise when searching $\Delta$ total min and $\Delta^{\prime}$ total min, By the formula (5) obtained optimum slope $\mathrm{K}$,

$$
\mathrm{K}=\left\{\mathrm{Ki} \mid \min \left\{\Delta \text { total } \min , \Delta^{\prime} \text { total } \min \right\}, \mathrm{i}=1,2, \ldots, 400\right\}
$$

$\mathrm{K}$ is the new coordinate system horizontal slope, By formula calculate the vertical axis slope -1 / $\mathrm{k}$, Then draw a new horizontal and vertical coordinates of the axes, calculate zero momentum of sight.

\section{Experimental and Results Analysis}

Under the program design, developed and installed an infrared sights zero momentum test system prototype.

The system uses the caliber $\Phi 200 \mathrm{~mm}$, off-axis parabolic mirror which focal length $1500 \mathrm{~mm}$ as mainly mirror, plane mirror to the secondary mirror combines reflective collimator, target pinhole diameter $\Phi 1 \mathrm{~mm}$ and blackbody band of $8 \sim 14 \mu \mathrm{m}$ together provide infinity infrared target to test system. Receive cross partition line and pinhole target image by CCD camera, transfer optical signal into an image signal, passed to the data processing system for processing

After infrared sights installed, gathering pinhole target and aiming partition image, marquee partition line, measured obtaining the center coordinates. Respectively marquee up, down, medium, left, right five round spots, After the camera posture correction adaptive algorithm to rebuild the reference coordinate system. Click the "coordinates" button, measured aiming partition coordinates in the reference coordinate system. Measuring time takes less than 20s, has high detection efficiency. After two sights chucking measured deviations shall be zero sights zero momentum. The camera posture corrected measurement results are shown in Table 1

Tab.1 Camera pose correction experiment data table

\begin{tabular}{ccccc}
\hline order & \multicolumn{3}{c}{$\begin{array}{c}\Delta \mathrm{X} \text { and } \triangle \mathrm{Y} \text { ranged from division to } \mathrm{X} \text { and } \mathrm{Y} \text { axis } \\
\text { When } C C D \text { camera is Tilted angle of } \alpha(\mathrm{mil}) .\end{array}$} \\
\cline { 2 - 5 } number & \multicolumn{3}{c}{$\alpha \approx 0^{\circ}$} & \multicolumn{3}{c}{$\alpha \approx 9^{\circ}$} \\
\cline { 2 - 5 } & $\mathrm{X}_{2}$ & $\mathrm{Y}_{2}$ & $\mathrm{X}_{1}$ & $\mathrm{Y}_{1}$ \\
\hline 1 & 4.903 & -2.122 & 4.900 & -2.119 \\
2 & 4.902 & -2.119 & 4.896 & -2.117 \\
3 & 4.902 & -2.119 & 4.897 & -2.116 \\
4 & 4.904 & -2.122 & 4.901 & -2.122 \\
5 & 4.902 & -2.120 & 4.903 & -2.119 \\
6 & 4.904 & -2.125 & 4.902 & -2.121 \\
\hline average & 4.902 & -2.121 & 4.899 & -2.119 \\
value & & & & \\
\hline & 0.0009 & 0.0021 & 0.0025 & 0.00208
\end{tabular}

Known from Table 1, when the CCD camera is no tilt and tilt $9^{\circ}$. In measuring coordinates aiming partition of the horizontal and vertical coordinates of the deviations were $0.003 \mathrm{mil}$ and $0.002 \mathrm{mil}$, and known by standard deviation the algorithm is good reproducibility, the results show, this paper presents an adaptive camera posture correction algorithm, can effectively avoid in measurement process since the camera tilt brings zero momentum measurement error.

Based on the measurement system prototype, using 0.5 "precision Leica theodolite instead CCD camera, alignment of the main axis of the infrared collimator, aim infrared sights aiming partition, read aiming partition coordinates in two locations-before and after assemble, calculate the $\mathrm{X}, \mathrm{Y}$ 
direction to move the zero momentum $\Delta \mathrm{X} 1, \Delta \mathrm{Y} 1$ be regarded as the standard value, and compares with $\Delta \mathrm{X} 2, \Delta \mathrm{Y} 2$ measured by the CCD camera, The measurement results are shown in Table 2 .

Tab.2 Zero momentum experiment data table

\begin{tabular}{ccccc}
\hline & \multicolumn{2}{c}{ Actual(mil) } & \multicolumn{2}{c}{ Calibration (mil) } \\
\cline { 2 - 5 } & $\Delta \mathrm{X} 1$ & $\Delta \mathrm{Y} 1$ & $\Delta \mathrm{X} 2$ & $\Delta \mathrm{Y} 2$ \\
\hline Value & 2.5156 & -1.1326 & 2.5045 & -1.1228 \\
\hline
\end{tabular}

Known by Table2, system detection accuracy in the horizontal and vertical directions respectively $0.0111 \mathrm{mil}$ and $0.0098 \mathrm{mil}$. The results showed that zero momentum accuracy is better than $0.02 \mathrm{mil}$.

\section{Conclusion}

Not involved in the measurement of the motion component in test system, neither need moving pinhole target, nor need achieve zero momentum measurement by rotating flat mirror, can avoid errors caused by movement parameters. Using camera posture adaptive correction algorithm, can avoid errors in measuring infrared sights zero momentum caused by camera tilt, detection accuracy is better than similar devices, to improve infrared sights zero momentum accuracy has important theoretical and practical value.

\section{References}

[1]Wang Jinsong, An Zhiyong,Li Hailan.Infrared reflective collimator sight zero momentum measurement method [J]. Acta Armamentar, 2010 (11): 1422 1425

[2]LI GUANG, WANG JINSONG,AN ZHIYONG. The Infrared Collimator Design for Infrared Sights Testing [J]. Journal of Changchun University of Science and Technology, 2011(4):71 73

[3]XIE BIN. Infrared sight zero momentum testing system[D]. Changchun University of Science and Technology, 2010.

[4]WEN BIN, LI YONGLIANG,ZENG YOUHONG. Research on the portable infrared sight baseline target detection system [J]. Laser and infrared, 2010, 40(012): 1330 1332.

[5]CHEN CONG, LU QIPENG, PENG ZHONGQI.Near infrared spectroscopy denoising method based on NLMS adaptive filter[J]. Acta Optica Sinica,2012,32(5):286-291.

[6]WANG WEIHUA,LI ZHIJUN,HE YAN etal.A real-time target detection algorithm for infrared search and track system based on region of interest extraction[J]. Chinese journal of lasers.2013,39(11):187-192. 